\title{
Caractéristiques des dépôts en réseau d'assainissement unitaire. Conséquences sur leur transport
}

\author{
O. Artières, G. Stotz \\ Institut für Siedlungswasserbau - Universität Stuttgart
}

\section{Introduction - Origine des dépôts}

Les dépôts stables et permanents accumulés dans les réseaux d'assainissement unitaires (ceux-ci évacuent à la fois les eaux usées domestiques et les eaux pluviales) posent depuis toujours de nombreux problèmes liés à leur présence : perturbation de l'écoulement, dégagement d'odeurs, accumulation de matières très polluantes, ... On remédiait jusqu'à présent à leurs inconvénients en organisant des campagnes de curage périodiques très fastidieuses et surtout fort coûteuses. Ce n'est que depuis peu qu'ils soulèvent des interrogations et que l'on cherche à s'en préoccuper plus activement.

Sur le cas d'un système d'assainissement d'une commune alsacienne, Entzheim, on a identifié l'origine des dépôts en recoupant les données issues d'une étude granulométrique précise et les résultats du bilan annuel entrée-sortie de matières solides dans le réseau : le dépôt stable est à $90 \%$ formé de particules minérales mélangées à de la matière organique domestique. Ces particules indésirables sont entraînées des surfaces extérieures par la lame d'eau ruissellante, et s'introduisent dans le réseau par les bouches d'égoût insuffisamment sélectives. La granulométrie passante est directement liée au type de bouche d'égoût en service sur le site. Une fois dans le réseau, ces particules s'y déposent, car les conditions hydrodynamiques préconisées dans les directives de calcul actuelles sont insuffisantes pour les transporter, notamment dans les grosses conduites [1]. Par une méthodologie différente, basée non pas sur une étude précise du phénomène, mais sur une observation statistique d'un grand nombre de cas, ВROM$\mathrm{BACH}$ [2] confirme et généralise cet afflux de particules minérales extérieures. Seule l'amélioration de l'efficacité des pièges à sédiment, que se doivent d'être les bouches d'égout, représente un moyen efficace et surtout durable de lutte contre les dépôts en réseau, car il s'applique à la cause du problème. Ce ne sera que par la suite que d'autres mesures d'accompagnement pourront être si nécessaire envisagées, comme par exemple l'étude de nouvelles valeurs limites de pose des canalisations.

Les dépôts encombrent malheureusement de nombreux réseaux, notamment dans les grosses agglomérations. Ces matériaux très pollués offrent des sites propices aux processus d'accumulation et de relargage d'éléments nocifs, tels que les métaux lourds [3]. Lorsque survient une crue pluviale, il se produit un transfert de matières polluantes des dépôts vers le fluide, soit par relargage de substances dissoutes dû à des conditions physico-chimiques différentes entre l'eau pluviale et l'eau usée de temps sec, soit par réentraînement d'une partie des dépôts pollués provoqué par l'accroissement des vitesses d'écoulement. C'est là un délicat problème de gestion des eaux pluviales et de protection de l'environnement qu'il s'agit de résoudre en acquérant une meilleure connaissance des caractéristiques de ce matériau particulier, ainsi que de leurs répercussions sur son transport.

\section{Characteristics of deposits in combined sewers. Consequences on their transport}

Deposits in combined sewers are constituted with sand particles from the streets and with organic domestic waste. Despite its small amount, this organic matter has a big influence on the deposit transport. It produces with the smaller silt particles a cohesive mixture that as the same mechanical properties as ton. This mixture binds the sand particles among themselves and increases their erosion strength. As many additional parameters that complicate the study of its transport and its modelisation. 


\begin{tabular}{|c|c|c|c|c|c|}
\hline $\begin{array}{c}\text { Type d'argile } \\
\text { ou de vase }\end{array}$ & $\begin{array}{c}\text { C.E.C. } \\
(\mathrm{meq} / 100 \mathrm{~g})\end{array}$ & $\begin{array}{l}W_{L} \\
\left(\%_{0}\right)\end{array}$ & $\begin{array}{l}W_{p} \\
(\%)\end{array}$ & IP & $\begin{array}{c}\text { Mat. Org. } \\
(\%)\end{array}$ \\
\hline Kaolinite & $3 \cdot 15$ & so & 25 & 25 & - \\
\hline Illite & 20.40 & 80 & 40 & 40 & - \\
\hline Montmorillonite & $80 \cdot 150$ & $100 \cdot 200$ & $40 \cdot 60$ & $60-140$ & - \\
\hline $\begin{array}{l}\text { Vase réseau } \\
\text { Entzheim }\end{array}$ & 20 & 87 & 44 & 43 & 22 \\
\hline $\begin{array}{l}\text { Vase réseau } \\
\text { Herrenberg }\end{array}$ & 34 & 80 & 45 & 35 & 14 \\
\hline
\end{tabular}

Tableau 1. Caractéristiques des vases argileuses et des vases de réseaux d'assainissement unitaires.

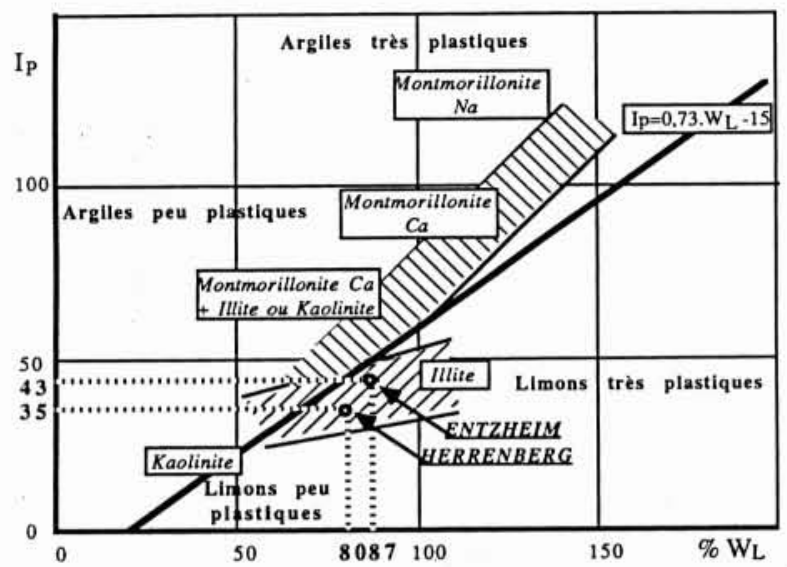

1. Points caractéristiques des vases de réseaux d'assainissement sur l'abaque de Casagrande.

Contrainte de cisaillement (en $\mathrm{N} / \mathrm{m}^{2}$ ).

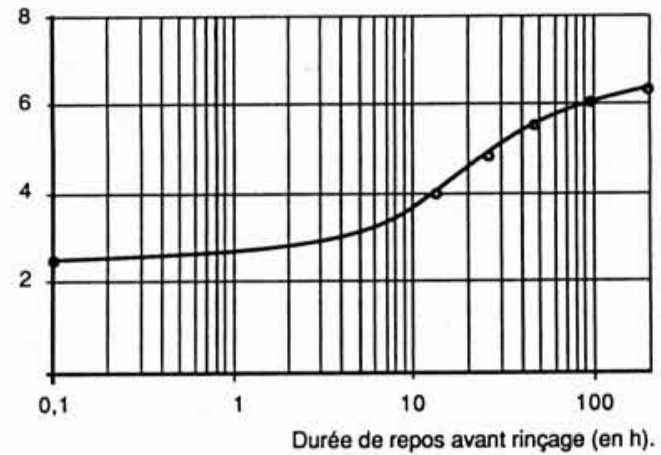

2. Contrainte critique de cisaillement en fonction de la durée de repos d'une boue primaire de station d'épuration.

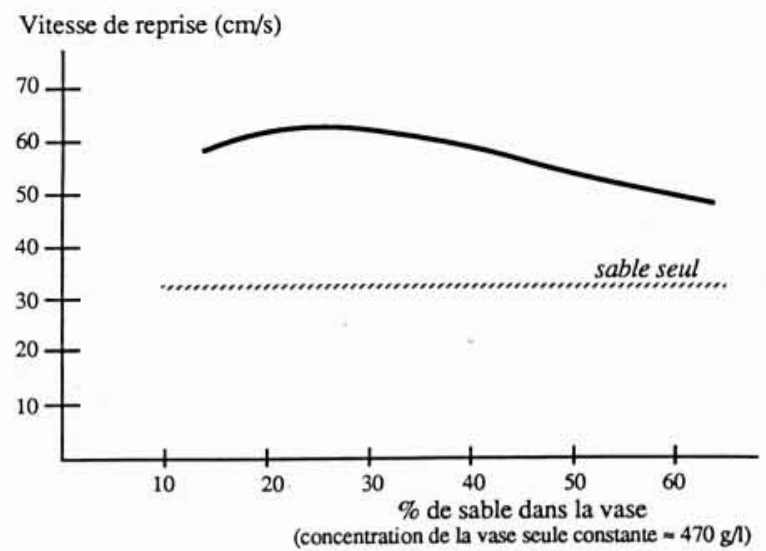

3. Influence de la teneur en sable d'un mélange sable-vase sur sa vitesse de reprise (d'après [5]).

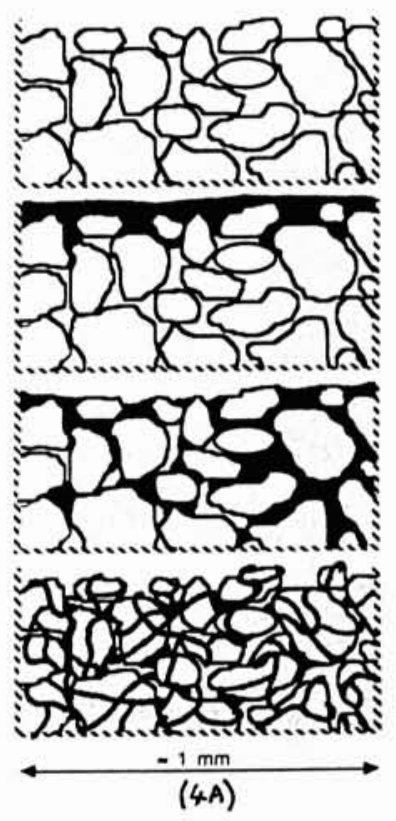

(a)

(b)

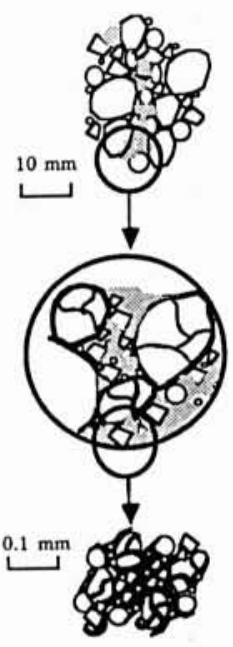

(4B)

4. Possibilité de processus de stabilisation biologique sur des granulométries uniforme $(4 A)$ et étendue $(4 B)$.

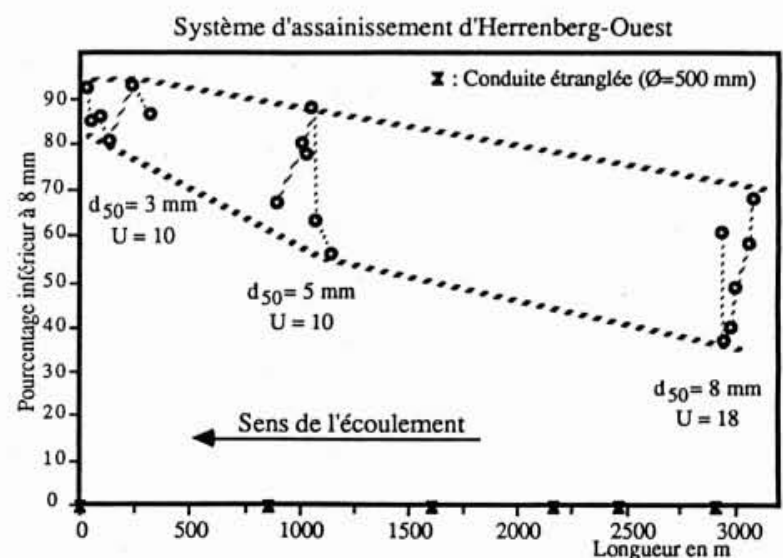

5. Tri granulométrique le long du collecteur d'Herrenberg.

Tableau 2. Comparaison de la vitesse de frottement de l'écoulement de temps sec avec la granulométrie du dépôt de réseau. 


\section{Structure et caractéristiques du dépôt}

La matière organique, mélangée aux particules minérales extérieures en proportions plus ou moins grandes selon le site, va considérablement modifier les caractéristiques de l'ensemble du dépôt: les particules colloïdes organiques (résultat de la décomposition des végétaux, substances précipitées, protėines, ...) ou biologiques (bactéries, ...) développent dans les eaux usées des charges électriques qui produisent des liaisons attractives de Van de Walls identiques à celles qui relient les micelles argileuses. La prédominance de ces forces attractives conduit au rapprochement des particules entre elles et à la formation de réseaux tridimensionnels de dimension très supérieure à celle des éléments qui les constituent. Ces réseaux organiques vont lier les particules minérales entre elles et augmenter leur résistance à l'érosion. Le mélange de la matière organique avec la plus fine fraction minérale $(<100 \mu \mathrm{m})$ produit une pâte cohésive noirâtre (vase) qui mastique les plus grosses particules sableuses entre elles. Cette cohésion peut s'approcher à l'aide de paramètres de nature, tels que limites d'Atterberg ou Capacité d'Echange en Cations. Bien que ne contenant pas d'argiles, ces paramètres révèlent que la vase de réseau a les mêmes caractéristiques mécaniques qu'une bonne argile de type Illite, et ceci indépendamment du site, puisque le collecteur intercommunal d'Herrenberg (RFA) a une capacité plus grosse que celui d'Entzheim (fig. 1, tabl. 1). Le réseau tridimensionnel organique se substitue donc de façon spectaculaire à celui formé par les micelles d'argile.

On retrouve également les propriétés de tassement et de consolidation mises en évidence pour les mixtures argileuses cohésives. La figure 2 montre par exemple l'importance de la durée de consolidation sur le transport de boues primaires [4]. En ce qui concerne le tassement, on observe en un mois le doublement de la concentration de la vase du réseau d'Entzheim. Des phénomènes parasites entravent cependant le bon déroulement du tassement (fermentation, décomposition) et nous rappellent que contrairement aux suspensions argileuses, il s'agit d'un matériau en perpétuelle évolution [3].

La proportion de matériau cohésif dans le dépôt aura une influence non négligeable sur son transport: plus le pourcentage de sable sera élevé, moins les liaisons intergrains seront parfaites, et moins les propriétés cohésives de la vase se feront sentir. Cependant, Migniot [4] note pour les vases argileuses, que malgré de forts pourcentages en sable $(60 \%)$, la vitesse de reprise du mélange sable-vase est encore majorée de $40 \%$ par rapport au sable seul (fig. 3).

Ces résultats sont également confirmés sur réseau d'assainissement. Malgré de faibles pourcentages dans le dépôt, l'effet cohésif de la vase organique est sensible. On s'en aperçoit en comparant la vitesse maximale de frottement de temps sec $U^{*}$ avec la taille des particules qui devraient être, grâce à cette vitesse, transportées en suspension, et donc a fortiori par charriage, s'il s'agissait de grains non cohésifs ( $t a b l .2)$. Ces vitesses correspondent au $d_{50}$ et au ${ }_{80}$ respectivement pour Herrenberg et Entzheim. Ainsi, si le sédiment était effectivement non cohésif, plus de la moitié du dépôt devrait être entraîné par temps sec. La différence entre les deux réseaux étudiés s'explique par des teneurs en vase différentes. Mais une faible teneur en vase comme à Herrenberg suffit à modifier le comportement du dépôt. Le transport des dépôts de réseau d'assainissement ne peut donc en aucune manière être traité comme s'il s'agissait de sable pur.

Ce phénomène de stabilisation organique d'un fond non cohésif n'est pas spécifique aux réseaux d'assainissement. Des études réalisées en région côtière [6] et en rivière [7] montrent l'influence du peuplement benthique et algual sur l'accroissement de stabilité du fond, à cause, par exemple, des mucosités et des glaires secrétées par certaines diatomées (augmentation de 2 à 5 fois de la vitesse critique d'érosion par rapport à ce qu'elle serait sans peuplement biologique). Le schéma de stabilisation biologique proposé par Führböter [6] s'adapte d'ailleurs très bien au réseau d'assainissement (fig. 4A) : en période de temps sec se forme à la surface du dépôt, une couche de matériau organique domestique dans laquelle se développe une activité bactérienne intense. Cette couche diminue la rugosité de surface, ce qui, pour un écoulement équivalent, occasionne une diminution du frottement sur le fond (b). L'échaffaudage biologique gagne en profondeur et cimente les grains entre eux, en remplissant partiellement ou complètement les pores (c), et renforce peu à peu le dépôt en étendant son réseau biologique tridimensionnel (d). Notons que ce mastiquage des pores est d'autant plus facile que la granulométrie est étendue, comme c'est le cas en réseau d'assainissement, où le coefficient d'uniformité $U=d_{60} / d_{10}$ est compris entre 8 et 20 (fig. $4 B$ ). Un tel échaffaudage biologique ne peut se développer que si les conditions hydrodynamiques de fond restent inférieures à la limite d'érosion, afin que le dépôt ne soit pas remanié sur une longue période. De telles conditions sont réunies soit en période de temps sec, car les vitesses y sont faibles, soit en période pluviale, lorsqu'il se produit des remous.

Malgré ces propriétés cohésives qui peuvent être plus ou moins marquées, on retrouve certains phénomènes observés en cours d'eau naturel avec du sédiment non cohésif. On constate d'une part un profil granulométrique à l'intérieur du dépôt, faisant apparaître des particules plus grossières en surface qu'au fond. Ce profil s'accompagne d'une teneur organique plus forte en profondeur. Ce phénomène rappelle le pavage en cours d'eau naturel. II montre en tout cas que l'écoulement peut laver la partie supérieure du dépôt, en entraînant à la fois la matière organique et les plus fines particules minérales, et en laissant sur place une couche protectrice de plus gros matériaux. Les écarts suivants ont été notés à Entzheim: $d_{50}=0,2 \mathrm{~mm}$ et teneur organique $=5 \%$ en surface, respectivement $0,05 \mathrm{~mm}$ et $30 \%$ au fond.

Un autre phénomène intéressant est le tri granulométrique observé le long de l'écoulement dans le collecteur d'Herrenberg, oủ le dépôt présente une granulométrie plus grossière en amont qu'en aval, qui s'accompagne d'un resserrement de la plage granulométrique ( $U$ passe de 18 à 10) (fig. 5) [8]. Le dépôt d'Entzheim plus riche en vase $(30 \%)$ ne fait par contre apparaître aucun tri. 


\section{Conclusion}

Au regard de son transport, le dépôt de réseau d'assainissement unitaire présente simultanément les caractéristiques liées à chacun de ses deux composants principaux : - d'une part, les propriétés cohésives et stabilisatrices transmises par la vase organique à l'ensemble du dépôt ; - d'autre part, les phénomènes plus classiques de pavage et de tri granulométrique dus à la matrice sableuse du dépôt.

La teneur en vase cohésive ressort comme étant un des facteurs déterminant de ce transport, qui suivant sa proportion dans le dépôt, privilégiera plus ou moins les phénomènes liés à la cohésion en masquant ceux liés aux particules sableuses, pourtant en quantité très supérieure.

Les caractéristiques des dépôts de réseau d'assainissement unitaire laissent ainsi présager la grande complexité de leur mode de transport, puisqu'au déjà très délicat problème du transport de sédiment non cohésif viennent s'ajouter de nouvelles variables. Sans oublier la méconnaissance actuelle de certains phénomènes hydrauliques en réseau (remous, poches d'air, turbulences aux jonctions) qu'il conviendra de maîtriser avant d'élaborer un bon modèle de prévision.

\section{Bibliographie}

[1] Artières, O. (1988), Peut-on éviter les dépôts en réseau d'assainissement unitaire? Techniques, Sciences et Méthodes, $n^{\circ}$ 9/88, p. 443-448.

[2] Вrombach, H. (1984), Einfluß des Einzugsgebietes auf die Rückhaltung von Schmutzfrachten an Regenüberlaufbecken. Schlußbericht, Bad Mergentheim, 80 p.

[3] Artières, O. (1987), Les dépôts en réseau d'assainissement unitaire. Origine, Caractéristiques, Pollution, Transport. Thèse de l'Univ. Louis-Pasteur de Strasbourg, 214 p.

[4] Krauth, Kh., G. Stotz (1985), Minimierung des Schmutzstoffeintrags aus Siedlungsgebieten in Vorfluter. Schlußbericht zum DFG-Forschungsvorhaben $\mathrm{Kr} 624 / 3-2$. Institut für Siedlungswasserbau, Universität Stuttgart, 233 p.

[5] Migniot, C. (1968), Etude des propriétés physiques de différents sédiments très fins et de leur comportement sous des actions hydrodynamiques. La Houille Blanche, Vol. 1, p. 591620 .

[6] FÜнRBÖteR, A. (1983), Über mikrobiologische Einflüsse auf den Erosionsbeginn bei Sandwatten. Wasser und Boden, $\mathrm{n}^{\circ} 3$, p. 106-116.

[7] SChröder, R.C.M., Chr. HeizelmanN (1987), Auswirkungen der biologischen Sohlenbesiedlung auf den Geschiebetransport in Fließgewässern. Wasser und Boden, $\mathrm{n}^{\circ}$ 11, p. 571-575.

[8] KRAUTH, Kh. (1988), Nutzung von Abwasserkanälen zur Leistungssteigerung des Klärsystems. 1. Statuskolloquium des Projektes Wasser-Abfall-Boden, Kernforschungszentrum, 23. Februar 1988, Karlsruhe, Deutschland.

[9] Bouvard, M. (1984), Barrages mobiles et ouvrages de dérivation, à partir de rivières transportant des matériaux solides. Collection de la Direction des Etudes et Recherches d'Electricité de France, Editions Eyrolles, Paris, 359 p. 